\title{
Refractive Outcomes of Uneventful Cataract Surgery in Pseudoexfoliation Syndrome and Pseudoexfoliation Glaucoma
}

\author{
Hatice Tekcan, Mehmet Serhat Mangan, Serhat Imamoglu, Oksan Alpogan \\ Department of Ophthalmology, Haydarpasa Numune Research and Training Hospital, Health Sciences University Turkey, Istanbul, \\ Turkey
}

Purpose: To compare the refractive outcomes after cataract surgery between patients with and without pseudoexfoliation, and to evaluate the risk factors of refractive error.

Methods: Retrospective review of 210 eyes with pseudoexfoliation (study group) and 510 normal eyes (control group) that underwent uneventful phacoemulsification with intraocular lens implantation. The study group included pseudoexfoliation syndrome and pseudoexfoliation glaucoma (PXG) subgroups. The main outcome measure was refractive error, defined as the difference between the target refraction and postoperative refraction in diopter (D). A refractive error $> \pm 1.0 \mathrm{D}$ was identified as a large-magnitude refractive error. The frequency of the large-magnitude refractive error was compared between study and control groups, and also between each subgroup and normal eyes. The factors influencing refractive error were analyzed by logistic regression.

Results: There was a significantly higher frequency of a refractive error $> \pm 1.0 \mathrm{D}$ and hyperopic error $>1.0 \mathrm{D}$ in the study group than in the control group ( $p=0.001$ and $p=0.003$, respectively). In the multivariate logistic regression analysis, PXG and poor preoperative visual acuity were related with a large-magnitude refractive error $(p=0.001$ and $p=0.02$, respectively) Refractive error $> \pm 1.0 \mathrm{D}$ and hyperopic error $>1.0 \mathrm{D}$ were noted more often in PXG eyes that had an intraocular pressure spike $>25 \mathrm{mmHg}$ at postoperative first 24 hours ( $p=0.01$ and $p=0.03$, respectively).

Conclusions: The eyes with pseudoexfoliation were at a high risk for refractive error, especially in the presence of glaucoma.

In PXG, the only significant risk factor for refractive error was observed to be the presence of an intraocular pressure spike.

Key Words: Cataract, Exfoliation glaucoma, Exfoliation syndrome, Intraocular pressure, Refractive errors

Pseudoexfoliation is the most common secondary cause of open-angle glaucoma and is associated with an in-

Received: December 2, 2021 Final revision: December 27, 2021 Accepted: January 22, 2022

Corresponding Author: Hatice Tekcan, MD. Department of Ophthalmology, Haydarpasa Numune Research and Training Hospital, Health Sciences University Turkey, Tibbiye Caddesi 40, Istanbul 34662, Turkey. Tel: 90505-765-0142, Fax: 90-216-278-2617, E-mail: drhatice.doner@gmail.com creased incidence of cataract $[1,2]$. Phacoemulsification can lead to significant intraocular pressure (IOP) reductions after surgery in pseudoexfoliation syndrome (PXS) and pseudoexfoliation glaucoma (PXG) [3,4]. Early postoperative IOP spikes are more common in eyes with pseudoexfoliation after phacoemulsification, especially in the presence of glaucoma [5].

The manifest change of the anterior chamber depth (ACD) have also been shown after phacoemulsification in 
eyes with PXS [6]. Pseudoexfoliation is related to zonular weakness. Weakening of the ciliary zonule may cause errors in refraction after intraocular lens (IOL) implantation even without complications such as rupture of the ciliary zonule and IOL subluxation [7]. However it is unclear how these changes might affect refractive outcome of phacoemulsification in eyes with pseudoexfoliation.

There is limited research about the effect of pseudoexfoliation on refractive outcomes after cataract surgery [8]. There has been no previous report comparing the incidence of refractive error after cataract surgery between PXG and PXS. The purpose of this study was to compare the refractive outcomes of uneventful cataract surgery between eyes with PXS and PXG and with normal eyes, in addition to determine the risk factors of refractive error after phacoemulsification with IOL implant.

\section{Materials and Methods}

\section{Ethics statement}

The study was approved by the Ethical Committee of Haydarpasa Numune Research and Training Hospital (No. HNEAH-KAK-KK-2020-6). The study protocol adhered to the tenets of the Declaration of Helsinki. A written consent was obtained from all patients who took part in this study.

\section{Study selection}

In this retrospective, case-control study, we reviewed the medical records of patients who underwent uneventful phacoemulsification with posterior chamber IOL implantation at a single center, between January 2015 and April 2020. The exclusion criteria were as follows: preexisting comorbid eye conditions that could affect the refractive outcomes (a history of trauma, corneal disease, retinal disease, and uveitis), previous ocular surgeries, intraoperative complications (capsule tear, vitreous loss, retained lens fragments, zonular dialysis, and choroidal haemorrhage), postoperative complications (prolonged corneal oedema, macular oedema, retinal detachment, and uncontrolled IOP requiring additional medication or glaucoma surgery within 6 months postoperatively), combined procedures such as phacoemulsification-trabeculectomy or phacoemulsifica- tion-pars plana vitrectomy and lack of visual acuity, IOP, and refractive data at postoperative 6-month visits. The eyes with axial length $\leq 22.0 \mathrm{~mm}$ and $\geq 26.0 \mathrm{~mm}$ and with preoperative cylindrical value $\geq 3.0$ diopters (D) were not included in the study. The control group included only those without pseudoexfoliation in both eyes.

The eyes that undergone uneventful cataract surgery with IOL implantation were classified into two groups. The study group including the eyes with pseudoexfoliation was categorized into two subgroups: PXS (eyes with pseudoexfoliation that did not have glaucomatous optic neuropathy and corresponding visual field defects) and PXG (eyes with pseudoexfoliation that had glaucomatous optic neuropathy and corresponding visual field defects). The normal eyes without pseudoexfoliation were included in the control group.

\section{Surgical technique}

All surgical procedures were performed under subtenon anesthesia. In all cases, a $2.8-\mathrm{mm}$ clear corneal incision in the upper corneal limbus, 5 to $6 \mathrm{~mm}$ continuous curvilinear capsulorrhexis, hydrodissection, and phacoemulsification with the Infiniti Vision System (Alcon Laboratories, Fort Worth, TX, USA) were performed to remove the cataract. A nonsutured capsular tension ring (CTR) was implanted in eyes with zonular weakness. A foldable onepiece acrylic hydrophobic IOL (Eyecryl Plus ASHFY600; Biotech Vision Care, Ahmedabad, India) with manufacturer-recommended A-constants of 118.5 was implanted in the capsular bag. All patients used prednisolone acetate and moxifloxacin drops during the postoperative first month.

The preoperative data included sex, age at phacoemulsification, IOP, corrected distance visual acuity (CDVA) as the logarithm of the minimum angle of resolution (logMAR), spherical equivalent (SE; spherical power $+1 / 2$ cylinder power), axial length (AL), IOL power (D), and predicted refraction. The measurement of refraction status was performed using an automatic refractor (Topcon KR800A Auto Refractor Keratometer; Topcon Medical, Tokyo, Japan). The IOL power was calculated using partial coherence interferometry (IOL Master 500; Carl Zeiss Meditec, Jena, Germany). The SRK/T formula was used to calculate the IOL power. 


\section{Outcome measures}

The primary outcome measure was the refractive error, defined as the difference in the predicted SE and postoperative SE at the 6-month visit. A refractive error $> \pm 1.0 \mathrm{D}$ was identified as a large-magnitude refractive error. The predictive refractive accuracy was analyzed, and the frequency of the refractive error was compared between eyes with and without pseudoexfoliation. The mean absolute error is defined as the absolute difference between the predicted SE and the actual postoperative SE. We also examined the difference in refractive outcomes between each subgroup and normal eyes. The mean IOP (average of two IOP measurements using Goldmann applanation tonometry), IOP change (difference between the preoperative and postoperative IOPs), and CDVA were recorded at 6-month

Table 1. Patient characteristics

\begin{tabular}{lccc}
\hline Characteristic & Study group & Control group & $p$-value \\
\hline No. of patients & 170 & 422 & - \\
No. of eyes & 210 & 510 & - \\
Age (yr) & $72.64 \pm 6.34$ & $72.13 \pm 5.11$ & $0.26^{*}$ \\
Sex & & & 219 \\
$\quad$ Male & 96 & 203 & - \\
Female & 74 & $23.37 \pm 0.85$ & $0.31^{\dagger}$ \\
Axial length (mm) & $23.32 \pm 0.79$ & $21.77 \pm 2.07$ & $0.53^{*}$ \\
Intraocular lens power (diopter) & $21.92 \pm 1.87$ & $0.38^{*}$ \\
\hline
\end{tabular}

Values are presented as number or mean \pm standard deviation; study group include eyes with pseudoexfoliation (pseudoexfoliation syndrome and pseudoexfoliation glaucoma); control group include normal eyes without pseudoexfoliation.

*Independent $t$-test; ${ }^{\circ}$ Pearson chi-square test.

Table 2. Summary of preoperative and postoperative descriptive statistics of study and control groups

\begin{tabular}{|c|c|c|c|}
\hline Variable & Study group & Control group & $p$-value \\
\hline No. of eyes & 210 & 510 & - \\
\hline Preoperative IOP, mean $\pm \mathrm{SD}(\mathrm{mmHg})$ & $16.43 \pm 3.24$ & $15.07 \pm 2.72$ & $<0.001^{*}$ \\
\hline Postoperative IOP, mean $\pm \mathrm{SD}(\mathrm{mmHg})$ & $13.67 \pm 3.04$ & $13.60 \pm 2.58$ & $0.73^{*}$ \\
\hline IOP change (mmHg) & & & $<0.001^{\dagger}$ \\
\hline Mean \pm SD & $-2.88 \pm 2.99$ & $-1.44 \pm 2.43$ & \\
\hline Median (range) & $-3.0(-8.0$ to -7.0$)$ & $-1.0(-8.0$ to -6.0$)$ & \\
\hline Preoperative CDVA (logMAR) & & & $0.79^{\dagger}$ \\
\hline Mean \pm SD & $0.74 \pm 0.47$ & $0.73 \pm 0.48$ & \\
\hline Median (range) & $0.52(0.22$ to 3.10$)$ & $0.70(0.15$ to 3.10$)$ & \\
\hline Postoperative CDVA (logMAR) & & & $<0.001^{\dagger}$ \\
\hline Mean \pm SD & $0.08 \pm 0.11$ & $0.04 \pm 0.08$ & \\
\hline Median (range) & $0.0(0.0$ to 0.7$)$ & $0.0(0.0$ to 1.0$)$ & \\
\hline Preoperative SE, mean $\pm \mathrm{SD}$ (diopter) & $-1.99 \pm 2.61$ & $-2.25 \pm 3.05$ & $0.39^{*}$ \\
\hline Postoperative SE, mean \pm SD (diopter) & $-0.40 \pm 0.77$ & $-0.52 \pm 0.59$ & $0.02^{*}$ \\
\hline
\end{tabular}

Study group include eyes with pseudoexfoliation (pseudoexfoliation syndrome and pseudoexfoliation glaucoma); control group include normal eyes without pseudoexfoliation.

$\mathrm{IOP}=$ intraocular pressure; $\mathrm{SD}=$ standard deviation; $\mathrm{CDVA}=$ corrected distance visual acuity; $\log \mathrm{MAR}=\operatorname{logarithm}$ of the minimum angle of resolution; $\mathrm{SE}=$ spherical equivalent.

*Independent $t$-test; ${ }^{\dagger}$ Mann-Whitney $U$-test. 
visit. In the first postoperative 24 hours, an IOP $>25 \mathrm{mmHg}$ was defined as an IOP spike $[9,10]$. If an IOP spike occurred, the treatment was $250 \mathrm{mg}$ of oral acetozolamid and injection of a $20 \%$ mannitol solution $(1 \mathrm{~g} / \mathrm{kg})$. There was no requirement of additional medical treatment or surgery after acute episode in the eyes with an IOP spike. The need for a CTR during cataract surgery was also recorded.

A statistical analysis was performed using SPSS ver. 16.0 (SPSS Inc., Chicago, IL, USA). Normality for continued variables in a group was determined using the Kolmogorov-Smirnov test. Descriptive analyses were represented as mean and standard deviation for a normal distribution or median and range for an abnormal distribution with a value of $p<0.05$. Groups were compared using the chi-square test or Fisher exact test for categorical variables and independent $t$-test or Mann-Whitney $U$-test for continuous variables. Independent predictors of the refractive error were determined by using binary logistic regression analy- sis. Patient age, sex, AL, preoperative CDVA, preoperative IOP, IOP change after phacoemulsification, PXS, PXG, CTR implantation during surgery, and presence of an IOP spike at postoperative first 24 hours were investigated as potential risk factors. First, each variable was analyzed in a univariate model. Next, all variables with a significance level of less than 0.20 were included in the multivariate model. A value of $p<0.05$ was considered statistically significant.

\section{Results}

A total of 592 patients (720 eyes) who underwent uneventful phacoemulsification cataract surgery met the inclusion criteria. We included 170 patients (210 eyes) with pseudoexfoliation as the study group, and 422 patients (510 eyes) without pseudoexfoliation as the control group. In the

Table 3. Mean refractive outcomes in the study and control groups

\begin{tabular}{lcccc}
\hline Variable & Mean refractive error $(\mathrm{D})$ & $p$-value & Mean absolute error (D) $^{*}$ & $p$-value \\
\hline Study group $(\mathrm{n}=210)$ & $0.08 \pm 0.77(-2.85$ to 2.10$)$ & 0.01 & $0.59 \pm 0.50(0.0$ to 2.85$)$ & 0.002 \\
PXS subgroup $(\mathrm{n}=82)$ & $0.08 \pm 0.67(-1.95$ to 1.47$)$ & 0.03 & $0.53 \pm 0.40(0.02$ to 1.95$)$ & 0.070 \\
PXG subgroup $(\mathrm{n}=128)$ & $0.08 \pm 0.83(-2.85$ to 2.10$)$ & 0.08 & $0.63 \pm 0.55(0.0$ to 2.85$)$ & 0.005 \\
Control group $(\mathrm{n}=510)$ & $-0.01 \pm 0.56(-1.42$ to 1.85$)$ & - & $0.45 \pm 0.35(0.0$ to 1.85$)$ & - \\
\hline
\end{tabular}

Values are presented as mean \pm standard deviation.

$\mathrm{D}=$ diopters; $\mathrm{PXS}=$ pseudoexfoliation syndrome; $\mathrm{PXG}=$ pseudoexfoliation glaucoma.

*Mann-whitney $U$-test (compared with control group).

Table 4. Rates of refractive errors in the study and control groups

\begin{tabular}{|c|c|c|c|c|}
\hline Variable & $\begin{array}{l}\text { Study group } \\
(\mathrm{n}=210)\end{array}$ & $\begin{array}{l}\text { PXS subgroup } \\
\quad(\mathrm{n}=82)\end{array}$ & $\begin{array}{l}\text { PXG subgroup } \\
\quad(\mathrm{n}=128)\end{array}$ & $\begin{array}{l}\text { Control group } \\
\quad(\mathrm{n}=510)\end{array}$ \\
\hline \multicolumn{5}{|l|}{ Refractive error } \\
\hline$> \pm 1.0 \mathrm{D}$ & $37(17.6)$ & $10(12.2)$ & $27(21.1)$ & $46(9.0)$ \\
\hline$p$-value ${ }^{*}$ & 0.001 & 0.36 & $<0.001$ & - \\
\hline \pm 0.5 to $\pm 1.0 \mathrm{D}$ & $55(26.2)$ & $27(32.9)$ & $28(21.9)$ & $132(25.9)$ \\
\hline$p$-value ${ }^{*}$ & 0.93 & 0.18 & 0.35 & - \\
\hline Hyperopic error $>+1.0 \mathrm{D}$ & $18(8.6)$ & $3(3.7)$ & $15(11.7)$ & $17(3.3)$ \\
\hline$p$-value ${ }^{*}$ & 0.003 & 0.54 & $<0.001$ & - \\
\hline Myopic error <-1.0 D & $19(9.0)$ & $7(8.5)$ & $12(9.4)$ & $29(5.7)$ \\
\hline$p$-value ${ }^{*}$ & 0.10 & 0.31 & 0.12 & - \\
\hline
\end{tabular}

Values are number of eyes presented as number (\%); study group include eyes with pseudoexfoliation $(\mathrm{n}=210 ; \mathrm{PXS}, \mathrm{n}=82 ; \mathrm{PXG}, \mathrm{n}=$ $128)$; control group include normal eyes without pseudoexfoliation $(n=510)$.

$\mathrm{PXS}=$ pseudoexfoliation syndrome; $\mathrm{PXG}=$ pseudoexfoliation glaucoma; $\mathrm{D}=$ diopters.

"Pearson chi-square test or Fisher exact test (compared with control group). 
study group, 70 patients (82 eyes) had a diagnosis of PXS, 100 patients (128 eyes) had a diagnosis of PXG. The patients' data are summarized in Table 1.

A summary of the descriptive statistics of the study and control eyes before (preoperative) and after (postoperative) cataract surgery with IOL implantation is reported in Table 2. There was statistically significant difference in terms of preoperative IOP, IOP change after phacoemulsification, and postoperative CDVA between two groups $(p<0.001)$. The postoperative CDVA $(0.13 \pm 0.33)$ was significantly worse in the eyes with PXG compared those with PXS $(0.05 \pm 0.69, p=0.01)$. There was no statistically significant difference for preoperative CDVA $(p=0.10)$, preoperative IOP $(p=0.08)$, postoperative IOP $(p=0.05)$, and IOP change $(p=0.70)$ in the subgroup analyses.

Tables 3 and 4 shows the refractive outcomes in the study and control groups. There was a significantly higher frequency of large-magnitude refractive and hyperopic errors in the study group, when compared with the control group ( $p=0.001$ and $p=0.003$, respectively). A large-magnitude refractive error and a hyperopic refractive error were also significantly more common in the eyes with PXG $(p<0.001)$ when compared to the control eyes. When we compared the refractive outcomes of PXG and PXS eyes with one another, only a hyperopic error was more frequent in PXG eyes $(p=0.03)$. There was no statistically significant difference in the mean refractive error and mean absolute error between PXG and PXS subgroups ( $p$ $=0.78$ and $p=0.56$, respectively).

Univariate logistic regression analysis of risk factors for refractive error $> \pm 1.0 \mathrm{D}$ in both study and control eyes was shown in Table 5. Preoperative CDVA, IOP spike, and PXG were related with refractive error $> \pm 1.0 \mathrm{D}$. These three factors were added in the multivariate model. PXS was also added in the multivariate model for subgroup analysis. Multivariate logistic regression analysis of risk factors for refractive error $> \pm 1.0 \mathrm{D}$ is shown in Table 6. PXG and low visual acuity before cataract surgery were found to be risk

Table 5. Univariate logistic regression analysis of risk factors for refractive error $> \pm 1.0$ diopters (720 eyes)

\begin{tabular}{lccc}
\hline Variable & Odds ratio & 95\% Confidence interval & -value $^{*}$ \\
\hline Age (yr) & 1.017 & $0.976-1.059$ & 0.41 \\
Sex & 1.285 & $0.756-2.182$ & 0.35 \\
Pseudoexfoliation syndrome & 1.075 & $0.531-2.175$ & 0.84 \\
Pseudoexfoliation glaucoma & 2.559 & $1.543-4.244$ & $<0.001$ \\
IOP spike & 2.245 & $1.205-4.180$ & 0.01 \\
Preoperative CDVA (logMAR) & 1.432 & $1.059-1.936$ & 0.02 \\
Preoperative IOP (mmHg) & 1.048 & $0.970-1.133$ & 0.23 \\
IOP change (mmHg) & 1.028 & $0.942-1.123$ & 0.53 \\
Axial length (mm) & 0.835 & $0.630-1.107$ & 0.21 \\
CTR implantation & 1.290 & $0.372-4.475$ & 0.68 \\
\hline
\end{tabular}

$\mathrm{IOP}=$ intraocular pressure; $\mathrm{CDVA}=$ corrected distance visual acuity; $\log \mathrm{MAR}=$ logarithm of the minimum angle of resolution; $\mathrm{CTR}=$ Capsule tension ring.

${ }^{*}$ Binary logistic regression analysis-univariate model; ${ }^{\dagger} \mathrm{IOP}>25 \mathrm{mmHg}$ at postoperative 24 hours.

Table 6. Multivariate logistic regression analysis of risk factors for refractive error $> \pm 1.0$ diopters (720 eyes)

\begin{tabular}{lccc}
\hline Variable & Odds ratio & 95\% Confidence interval & $p$-value \\
\hline Preoperative CDVA (logMAR) & 1.437 & $1.055-1.959$ & 0.020 \\
Pseudoexfoliation syndrome & 1.307 & $0.627-2.721$ & 0.470 \\
Pseudoexfoliation glaucoma & 2.546 & $1.488-4.356$ & 0.001 \\
Intraocular pressure spike $^{\dagger}$ & 1.776 & $0.928-3.397$ & 0.080 \\
\hline
\end{tabular}

$\mathrm{CDVA}=$ corrected distance visual acuity; $\log \mathrm{MAR}=$ logarithm of the minimum angle of resolution.

*Binary logistic regression analysis-univariate model; ${ }^{\dagger}$ Intraocular pressure $>25 \mathrm{mmHg}$ at postoperative 24 hours. 
Table 7. Analysis of risk factors for refractive error $> \pm 1.0 \mathrm{D}$ in pseudoexfoliation glaucoma

\begin{tabular}{|c|c|c|c|}
\hline Risk factor & $\begin{array}{c}\text { Refractive error } \\
> \pm 1.0 \mathrm{D}(\mathrm{n}=27)\end{array}$ & $\begin{array}{l}\text { No refractive error } \\
> \pm 1.0 \mathrm{D}(\mathrm{n}=101)\end{array}$ & $p$-value \\
\hline Sex (no. of patients) & & & $0.36^{*}$ \\
\hline Male & 15 & 48 & \\
\hline Female & 16 & 31 & \\
\hline Age (yr) & $72.03 \pm 6.35$ & $72.26 \pm 6.33$ & $0.86^{\dagger}$ \\
\hline$>70(\mathrm{n}=80,63 \%)$ & $15(56)$ & $65(64)$ & $0.40^{*}$ \\
\hline$\leq 70(\mathrm{n}=48,37 \%)$ & $12(44)$ & $36(36)$ & \\
\hline Glaucoma duration (mon) & $67.40 \pm 9.36$ & $42.30 \pm 4.42$ & $0.05^{\dagger}$ \\
\hline Preoperative CDVA (logMAR) & $0.87 \pm 0.60$ & $0.79 \pm 0.63$ & $0.52^{\dagger}$ \\
\hline$\geq 1.0(\mathrm{n}=43,34 \%)$ & $12(44)$ & $31(31)$ & $0.17^{*}$ \\
\hline$<1.0(\mathrm{n}=85,66 \%)$ & $15(56)$ & $70(69)$ & \\
\hline Preoperative IOP (mmHg) & $16.80 \pm 2.60$ & $16.60 \pm 3.10$ & $0.79^{\dagger}$ \\
\hline IOP change $(\mathrm{mmHg})$ & $-2.10 \pm 3.40$ & $-2.90 \pm 2.80$ & $0.24^{\dagger}$ \\
\hline Axial length (mm) & $23.40 \pm 0.90$ & $23.23 \pm 0.73$ & $0.29^{\dagger}$ \\
\hline$<23(\mathrm{n}=52,41 \%)$ & $11(41)$ & $41(41)$ & $0.98^{*}$ \\
\hline $23-25(\mathrm{n}=72,56 \%)$ & $14(52)$ & $58(57)$ & $0.60^{*}$ \\
\hline$>25(\mathrm{n}=4,3 \%)$ & $2(7)$ & $2(2)$ & $0.19^{*}$ \\
\hline IOP spike & & & $0.01^{*}$ \\
\hline Yes $(n=25,20 \%)$ & $10(37)$ & $15(15)$ & \\
\hline No $(n=103,80 \%)$ & $17(63)$ & $86(85)$ & \\
\hline Capsule tension ring & & & $0.69^{*}$ \\
\hline Yes $(n=12,9.4 \%)$ & $2(7)$ & $10(10)$ & \\
\hline No $(\mathrm{n}=116,90.6 \%)$ & $25(93)$ & $91(90)$ & \\
\hline
\end{tabular}

Values are presented as number only, mean \pm standard deviation, or number $(\%)$; numbers are number of eyes unless otherwise indicated.

$\mathrm{D}=$ diopters; $\mathrm{CDVA}=$ corrected distance visual acuity; $\log \mathrm{MAR}=$ logarithm of the minimum angle of resolution; IOP=Intraocular pressure.

*Pearson chi-square test or Fisher exact test; ${ }^{\dagger}$ Independent $t$-test; ${ }^{\star} \mathrm{IOP}>25 \mathrm{mmHg}$ at postoperative 24 hours.

Table 8. Univariate logistic regression analysis of risk factors for refractive error $> \pm 1.0$ diopters in the pseudoexfoliation glaucoma subgroup (128 eyes)

\begin{tabular}{lccc}
\hline Variable & Odds ratio & 95\% Confidence interval & $p^{\text {-value }}{ }^{*}$ \\
\hline Age (yr) & 0.994 & $0.929-1.064$ & 0.86 \\
Sex & 0.619 & $0.217-1.768$ & 0.37 \\
IOP spike $^{\dagger}$ & 3.373 & $1.299-8.759$ & 0.01 \\
Preoperative CDVA (logMAR) & 1.230 & $0.654-2.315$ & 0.52 \\
Preoperative IOP (mmHg) & 1.019 & $0.885-1.173$ & 0.79 \\
IOP change (mmHg) & 1.085 & $0.946-1.245$ & 0.24 \\
Axial length (mm) & 1.333 & $0.777-2.286$ & 0.29 \\
CTR implantation & 0.728 & $0.150-3.539$ & 0.69 \\
\hline
\end{tabular}

$\mathrm{IOP}=$ intraocular pressure; $\mathrm{CDVA}=$ corrected distance visual acuity; $\log \mathrm{MAR}=$ logarithm of the minimum angle of resolution; $\mathrm{CTR}=$ capsule tension ring.

${ }^{*}$ Binary logistic regression analysis-univariate model; ${ }^{\dagger} \mathrm{IOP}>25 \mathrm{mmHg}$ at postoperative 24 hours. 
factor for large-magnitude refractive error ( $p=0.001$ and $p=0.02$, respectively).

In the study group, CTR was implanted in 12 eyes with PXG (9.4\%) and seven eyes with PXS (8.5\%). In the control group, CTR was implanted in only two eyes $(0.4 \%)$. There was no statistically significant relationship between large-magnitude refractive error and CTR implantation in the PXG and PXS subgroups and in the control group ( $p=0.51, p=0.61$, and $p=0.82$, respectively).

Table 7 shows a comparison of risk factors between the eyes with and without large-magnitude refractive error in PXG eyes. At postoperative 24 hours, an IOP spike was seen in 37 normal eyes (7.3\%) compared to 25 eyes (19.5\%) with PXG $(p<0.001)$ and 10 eyes $(12.2 \%)$ with PXS $(p=0.12)$. In the PXG subgroup, the eyes with refractive error $> \pm 1.0 \mathrm{D}$ and hyperopic error $>1.0 \mathrm{D}$ after cataract surgery, had a significantly higher frequency of IOP spikes than those with no refractive error $(p=0.01$ and $p=0.03$, respectively). Using logistic regression analysis, the odds ratio of an IOP spike was 3.37 (95\% confidence interval, $1.29-8.75)$, which was statistically significant for a large-magnitude refractive error $(p=0.01)$ (Table 8$)$.

\section{Discussion}

Despite advances in technology involving phacoemulsification and IOL design, refractive outcomes of cataract surgery are not always perfect. Parameters, determining the IOL power to be used, include the lens constant provided by the manufacturer, keratometry measurements, ACD, and the AL of the eye [11]. The SRK/T is a third generation theoretical formula that uses a modified ACD prediction algorithm [12]. This formula calculates estimation of IOL position by considering the AL and corneal height.

This is the first study with a large series of refractive outcomes of eyes with pseudoexfoliation (210 eyes) and is the first comparison of the refractive errors after cataract surgery in patients with PXS and PXG. Our results showed that there was a significant difference in large-magnitude refractive error $(> \pm 1.0 \mathrm{D})$ and hyperopic error $(>1.0 \mathrm{D})$ between eyes with and without pseudoexfoliation. Although there was no significant difference for each refractive error between PXS and normal eyes, in PXG eyes, large-magnitude refractive and hyperopic errors were significantly more frequent than in normal eyes. In addition, the inci- dence of a hyperopic error was significantly higher in PXG eyes than in PXS eyes.

There have been a few studies describing the refractive outcomes after cataract surgery in eyes with pseudoexfoliation. Ishikawa et al. [8] observed 62 eyes with pseudoexfoliation. They reported that in eyes with pseudoexfoliation, the SE refraction errors were distributed more widely than in normal eyes in both myopic and hyperopic directions on the postoperative first day. There was no significant intergroup difference in refractive error at postoperative month 1 . However, the patients were not clearly defined as PXS or PXG. In a study by Gur Gungor et al. [6], PXS $(n=22)$ and normal eyes $(n=30)$ were compared for refractive outcomes and ACD changes after uneventful phacoemulsification. At postoperative month 3 , the change in ACD was significantly larger in PXS eyes than in normal eyes. The mean absolute refractive error calculated using different IOL formulas (SRK/T, Haigis, Hoffer, and Holladay 1) was not statistically significant between the two groups. In the present study, 82 eyes with PXS had no significant frequency of refractive error when we compared with normal eyes, consistent with other recent studies.

Several studies have reported significant deepening of the anterior chamber with a reduced IOP after phacoemulsification in normal eyes $[13,14]$. They found that the decrease in IOP did not correlate with the degree of increase in the ACD. In our study, the decrease in IOP was significantly greater in eyes with pseudoexfoliation than in eyes without pseudoexfoliation, and this difference in IOP change did not correlate with the refractive outcomes.

Ucakhan et al. [15] and Kim et al. [16] reported that the shallower the preoperative anterior chamber, the deeper it became postoperatively. Ning et al. [17] indicated that a hyperopic or myopic shift often occurs with different degrees of change in the ACD. Muzyka-Wozniak and Ogar [18] found that the relative change in the ACD was larger in short eyes than in normal eyes or long eyes, which means that the $\mathrm{AL}$ influenced changes on the postoperative ACD.

Only one study has evaluated anterior segment parameters in PXG and PXS eyes. They found that there was a significant difference in means of ACD values, compared to normal eyes in the PXG group but not in the PXS group [19]. In the Yang et al. [20]'s study, hyperopic shift existed in age-related cataract patients with shallow anterior cham- 
ber and the shallower of ACD was, the greater of hyperopic shift happened. There were significantly more frequent hyperopic refractive errors in PXG eyes than in PXS and normal eyes in our study. Hyperopic error could be explained by a preoperative shallower $\mathrm{ACD}$ and postoperative higher increase in ACD in PXG eyes, when compared with PXS and normal eyes.

Several risk factors for deviating from the predicted postoperative refraction have been reported. These include older age, sex, glaucoma, poor preoperative visual acuity, previous eye surgery, surgical complications, AL measurement, and estimation of IOL position [21-23]. They suggested that a measurement error in biometry and keratometry in patients with low preoperative visual acuity could negatively affect refractive outcomes after cataract surgery. The subtypes of glaucoma and the myopic or hyperopic refractive error were not specified in this study. In the present study, poor visual acuity before cataract surgery was related with refractive error. Manoharan et al. [24] reported that primary open-angle glaucoma, PXG, and chronic angle-closure glaucoma eyes had higher odds for large-magnitude refractive errors than eyes without glaucoma, but only PXG and chronic angle-closure glaucoma eyes were statistically significant. Until our study, it was the first study that analyzed PXG (23 eyes) as a risk factor for refractive error after cataract surgery. Our study included a larger case series for PXG group $(n=128)$ that showed higher odds of a large-magnitude refractive error and hyperopic error when compared with PXS and normal eyes.

In our study, there was no statistically significant relationship between large-magnitude refractive error and CTR implantation in the PXG and PXS subgroups and in the control group. This result was similar to previous publications $[25,26]$.

Yeh et al. [27] showed that an IOP spike of $>50 \%$ above the baseline IOP may contribute to a myopic condition in the low preoperative IOP $(<9 \mathrm{mmHg})$ group in the trabeculectomized eyes. They suggested that a period of higher IOP after phacoemulsification stretches the eye, resulting in a longer $\mathrm{AL}$ and induction of a myopic shift. Lee et al. [28] reported a significant relationship between unpredictable refractive outcomes and acute angle-closure glaucoma duration in primary angle-closure glaucoma patients. They suggested that the longer the duration of IOP elevation, the greater the likelihood that zonular instability will occur, so in patients with a longer acute angle-closure glaucoma duration could explain why unpredictable refractive outcomes frequently develop in these patients after cataract surgery. In the present study, the IOP spike was more common in the PXG subgroup (20\%). The only significant predictive factor, found in our study related to large-magnitude refractive error and hyperopic error, was the presence of an IOP spike in PXG eyes. A hyperopic shift in ocular power occurs when an IOL is implanted in a more posterior plane than preoperatively planned [29]. An IOP spike may cause the postoperative ACD to be deeper than predicted, with preexisting zonular instability. It could explain why a hyperopic refractive error was more frequently seen in PXG eyes with an IOP spike.

As a limitation we could not observe the keratometry values, $\mathrm{ACD}$, and $\mathrm{AL}$ changes after phacoemulsification because of the retrospective nature of the study. Also, IOL power was calculated with SRK/T, so we could not draw any conclusions about the influence of different IOL calculations on the refractive outcome. There is a need for additional prospective and randomized studies to evaluate the relationship between refractive outcomes and changes in anterior chamber parameters after cataract surgery in PXG eyes.

In conclusion, in the present study, we observed that the eyes with PXG but not PXS were at high risk for refractive error, particularly hyperopic shift. The presence of an IOP spike was related to large-magnitude refractive error and hyperopic error in PXG eyes. In PXG, the cause of refractive surprises after uneventful cataract surgery, may be related to unpredictable ACD changes in the presence of zonular weakness.

Conflicts of Interest: None.

Acknowledgements: None.

Funding: None.

\section{References}

1. Hiller R, Sperduto RD, Krueger DE. Pseudoexfoliation, intraocular pressure, and senile lens changes in a population-based survey. Arch Ophthalmol 1982;100:1080-2.

2. Puska P, Tarkkanen A. Exfoliation syndrome as a risk factor for cataract development: five-year follow-up of lens opacities in exfoliation syndrome. J Cataract Refract Surg 
2001;27:1992-8

3. Merkur A, Damji KF, Mintsioulis G, Hodge WG. Intraocular pressure decrease after phacoemulsification in patients with pseudoexfoliation syndrome. J Cataract Refract Surg 2001;27:528-32.

4. Shingleton BJ, Laul A, Nagao K, et al. Effect of phacoemulsification on intraocular pressure in eyes with pseudoexfoliation: single-surgeon series. J Cataract Refract Surg 2008; 34:1834-41.

5. Mierzejewski A, Eliks I, Kaluzny B, et al. Cataract phacoemulsification and intraocular pressure in glaucoma patients. Klin Oczna 2008;110:11-7.

6. Gur Gungor S, Akman A, Asena L, et al. Changes in anterior chamber depth after phacoemulsification in pseudoexfoliative eyes and their effect on accuracy of intraocular lens power calculation. Turk J Ophthalmol 2016;46:255-8.

7. Masket S, Osher RH. Late complications with intraocular lens dislocation after capsulorhexis in pseudoexfoliation syndrome. J Cataract Refract Surg 2002;28:1481-4.

8. Ishikawa N, Hayashi Y, Miyamoto T, Saika S. Errors in the prediction of postoperative refraction following intraocular lens implantation in eyes with pseudoexfoliation syndrome. $J$ Cataract Refract Surg 2013;39:649-50.

9. Chen PP, Weaver YK, Budenz DL, et al. Trabeculectomy function after cataract extraction. Ophthalmology 1998;105: 1928-35.

10. Hayashi K, Yoshida M, Sato T, et al. Intraocular pressure elevation after cataract surgery and its prevention by oral acetazolamide in eyes with pseudoexfoliation syndrome. $J$ Cataract Refract Surg 2018;44:175-81.

11. Aristodemou P, Knox Cartwright NE, et al. Formula choice: Hoffer Q, Holladay 1, or SRK/T and refractive outcomes in 8108 eyes after cataract surgery with biometry by partial coherence interferometry. J Cataract Refract Surg 2011;37:63-71.

12. Olsen T. Calculation of intraocular lens power: a review. Acta Ophthalmol Scand 2007;85:472-85.

13. Altan C, Bayraktar S, Altan T, et al. Anterior chamber depth, iridocorneal angle width, and intraocular pressure changes after uneventful phacoemulsification in eyes without glaucoma and with open iridocorneal angles. $J$ Cataract Refract Surg 2004;30:832-8.

14. Doganay S, Bozgul Firat P, Emre S, Yologlu S. Evaluation of anterior segment parameter changes using the Pentacam after uneventful phacoemulsification. Acta Ophthalmol 2010;88:601-6.
15. Ucakhan OO, Ozkan M, Kanpolat A. Anterior chamber parameters measured by the Pentacam CES after uneventful phacoemulsification in normotensive eyes. Acta Ophthalmol 2009;87:544-8.

16. Kim M, Park KH, Kim TW, Kim DM. Changes in anterior chamber configuration after cataract surgery as measured by anterior segment optical coherence tomography. Korean J Ophthalmol 2011;25:77-83.

17. Ning X, Yang Y, Yan H, Zhang J. Anterior chamber depth: a predictor of refractive outcomes after age-related cataract surgery. BMC Ophthalmol 2019;19:134.

18. Muzyka-Wozniak M, Ogar A. Anterior chamber depth and iris and lens position before and after phacoemulsification in eyes with a short or long axial length. J Cataract Refract Surg 2016;42:563-8.

19. Doganay S, Tasar A, Cankaya C, et al. Evaluation of Pentacam-Scheimpflug imaging of anterior segment parameters in patients with pseudoexfoliation syndrome and pseudoexfoliative glaucoma. Clin Exp Optom 2012;95:218-22.

20. Yang F, Hou X, Wu H, Bao Y. Analysis of refractive status after cataract surgery in age-related cataract patients with shallow anterior chamber. Zhonghua Yan Ke Za Zhi 2014; 50:84-8.

21. Kugelberg M, Lundstrom M. Factors related to the degree of success in achieving target refraction in cataract surgery: Swedish National Cataract Register study. J Cataract Refract Surg 2008;34:1935-9.

22. Norrby S. Sources of error in intraocular lens power calculation. J Cataract Refract Surg 2008;34:368-76.

23. Lundstrom M, Dickman M, Henry Y, et al. Risk factors for refractive error after cataract surgery: analysis of 282811 cataract extractions reported to the European Registry of Quality Outcomes for cataract and refractive surgery. $J$ Cataract Refract Surg 2018;44:447-52.

24. Manoharan N, Patnaik JL, Bonnell LN, et al. Refractive outcomes of phacoemulsification cataract surgery in glaucoma patients. J Cataract Refract Surg 2018;44:348-54.

25. Schild AM, Rosentreter A, Hellmich M, et al. Effect of a capsular tension ring on refractive outcomes in eyes with high myopia. J Cataract Refract Surg 2010;36:2087-93.

26. Saadet GI, Fatih O. Effect of the capsular tension ring on refractive outcome after phacoemulsification. Rom J Ophthalmol 2021;65:59-63.

27. Yeh OL, Bojikian KD, Slabaugh MA, Chen PP. Refractive outcome of cataract surgery in eyes with prior trabeculectomy: risk factors for postoperative myopia. J Glaucoma 
H Tekcan, et al. Refractive Outcomes of Cataract Surgery in Pseudoexfoliation

2017;26:65-70.

28. Lee HS, Park JW, Park SW. Factors affecting refractive outcome after cataract surgery in patients with a history of acute primary angle closure. Jpn J Ophthalmol 2014;58:33-9.
29. Erickson P. Effects of intraocular lens position errors on postoperative refractive error. J Cataract Refract Surg 1990;16:305-11. 